\title{
Seletividade alimentar: uma abordagem nutricional
}

\author{
Selective eating: a nutritional approach \\ Ana Beatriz de Mello Sampaio', Thais Lourenço Nogueira', Ruth Bartelli Grigolon', Ana Maria Roma', \\ Leticia Enrique Pereira', Karin Louise Lenz Dunker'
}

\section{Palavras-chave}

Seletividade, alimentação, avaliação nutricional.

\section{Keywords}

Selective, eating, evaluation nutritional.

\section{RESUMO}

A seletividade alimentar é caracterizada por recusa alimentar, pouco apetite e desinteresse pelo alimento. É um comportamento típico da fase pré-escolar, mas, quando presente em ambientes familiares desfavoráveis, pode acentuar-se e permanecer até a adolescência. Este artigo trata de um relato de caso em que o paciente, com diagnóstico de seletividade alimentar, inicia tratamento em serviço especializado de transtornos alimentares aos 14 anos. A particularidade deste caso é a rápida e boa evolução do quadro, possivelmente decorrente do desejo próprio de se tratar e do apoio recebido pela família. A análise do caso em questão aponta para a importância de identificar os casos de seletividade de forma correta e precoce para que eles sejam encaminhados o quanto antes a profissionais habilitados no tratamento de distúrbios alimentares nos diferentes estágios de desenvolvimento da infância e adolescência, resultando em melhor prognóstico do quadro.

\section{ABSTRACT}

Selective eating is known by food refusal, lack of appetite and interest in food. It is atypical behavior of preschool, but when present in adverse family environments, can perpetuate and remain until adolescence. This paper is a case report of a patient with diagnosis of selective eating that seeks for specialized treatment center for eating disorders at 14 years old. The particularity of this case is the fast and good evolution, possibly due to the desire to treat himself and the support received by the family. The case points out the importance of identifying earlier and correctly cases of selectivity so they will be submitted as soon as possible to professionals specialized in treatment of eating disorders in different developmental stages of childhood and adolescence, resulting in a better prognosis framework. 


\section{INTRODUÇÃO}

A recusa alimentar é um comportamento típico da primeira infância, caracterizado por comportamentos como: fazer birras, demorar a comer, tentar negociar o alimento que será consumido, levantar da mesa durante a refeição e beliscar ao longo do dia. No entanto, parece haver crianças que persistem com comportamentos peculiares até meados da infância ou continuam pelas demais fases da vida. Esses comportamentos seriam definidos como seletividade alimentar (SA), que é caracterizada por um consumo alimentar altamente limitado e extrema resistência em experimentar novos alimentos. Esse tipo de comportamento resulta em uma limitação das atividades sociais relacionadas à alimentação ${ }^{1,2}$.

De acordo com os sistemas de classificação DSM-IV e CID10, a SA não está descrita como um diagnóstico específico de transtorno alimentar na infância, mas ambos associam-na a uma dificuldade persistente em comer adequadamente, com falha no ganho de peso ou importante perda ponderal durante o último mês. Não existem condições fisiopatológicas ou desordens mentais associadas, não há falta de acesso aos alimentos e o distúrbio deve ter início antes dos 6 anos ${ }^{1,2}$.

Os dados de prevalência de SA são escassos, mas vêm sendo caracterizados nas percepções e relatos de pais e cuidadores. Segundo eles, a seletividade seria mais frequente em crianças de 4 a 24 meses (19\% a 50\% dos casos) $)^{3}$. A imprecisão sobre a definição exata da SA, assim como a variabilidade de critérios metodológicos utilizados nos estudos, dificulta a determinação da prevalência. Não há evidências que relacionem o quadro de SA com comportamentos característicos de transtornos alimentares, como: fazer dieta, episódios de compulsões alimentares e controle obsessivo do peso corporal ${ }^{2}$. A ausência de dados consistentes de prevalência, definição do distúrbio e de suas características, principalmente em termos das escolhas alimentares, justifica a descrição do relato de caso de seletividade alimentar, fornecendo informações que podem auxiliar profissionais da área da saúde na identificação e no tratamento do distúrbio.

\section{METODOLOGIA}

O caso foi escolhido considerando a evolução nutricional positiva. Os dados foram coletados no prontuário clínico e nutricional.

A avaliação da dieta quanto à qualidade e quantidade foi realizada por meio do recordatório alimentar de $24 \mathrm{~h}$ e sua evolução, acompanhada por meio do Registro Alimentar. Os valores nutricionais foram calculados por meio do programa de análise nutricional (Avanutri) e comparados com os padrões recomendados pelo National Research Council (NRC) e Standing Committee on the Scientific Evaluation of Dietary Reference Intakes (DRI Committee) para valor calórico total, porcentagem de proteínas, carboidratos, gorduras, além do consumo de cálcio, ferro e vitamina A.

Utilizou-se a balança digital Filizola com estadiômetro e capacidade máxima de 200 kg para aferição de peso e estatura. O estado nutricional foi avaliado a partir do índice de massa corporal (IMC) e analisado por meio das curvas de crescimento da Organização Mundial da Saúde (2006/2007) para estatura e IMC para idade e sexo. Consideram-se normalidade os valores encontrados entre o P3 e P85. Nesse caso, o traçado preto indica antes e o cinza, durante o tratamento nutricional (vide legenda nas Figuras 1 e 2).

O tratamento foi realizado em serviço público especializado (hospital/escola), sendo parte do protocolo o Termo de Consentimento Livre e Esclarecido dos pacientes.

\section{RELATO DE CASO}

Encaminhado para tratamento especializado em transtornos alimentares, VNS é um paciente de 14 anos, sexo masculino, natural de São Paulo-SP, estudante da nona série do ensino fundamental, apresenta bom desempenho escolar e mora com os pais e um irmão de 9 anos.

A avaliação psiquiátrica indicou a queixa principal de SA com prejuízo na vida social. Observou-se ausência de tristeza e de sintomas psicóticos, compulsão alimentar ou desconforto com o peso e forma corporal, mas demonstrou preocupação com o crescimento físico. Relatou que desde os 10 anos de idade sente-se desconfortável ao comer diante de outras pessoas, sugerindo um diagnóstico de fobia social. Foi prescrita sertralina $25 \mathrm{mg} /$ dia inicialmente com aumento posterior, até se alcançar a dose de $50 \mathrm{mg} /$ dia, para melhorar o comportamento social e facilitar o convívio com os amigos. O paciente foi encaminhado para acompanhamento nutricional na Instituição e sugeriu-se acompanhamento psicológico com abordagem em terapia cognitivo-comportamental (TCC).

O primeiro atendimento nutricional incluiu uma investigação sobre a história alimentar do paciente desde o seu nascimento: o aleitamento materno exclusivo ocorreu por apenas 5 dias; as frutas foram incluídas na alimentação aos 5 meses e a sopa liquidificada, aos 6 meses, permanecendo como parte da dieta até 1 ano de idade.

Desde então iniciou recusa da sopa e de novos alimentos oferecidos pela mãe e manteve somente o consumo de leite com Sustagem ${ }^{\oplus}$, sucos e frutas. A mãe ofereceu vários alimentos em diversas formas de apresentação e, após muitas tentativas, ele aceitou cebola em rodelas e batata frita palito, o que demonstra preferência por alimentos de cor clara, sendo esta a base de suas refeições durante a infância. Com o tempo, estabeleceu um cardápio próprio que incluiu batata smile assada, biscoitos, pães, salgadinhos industrializados, leite integral, refrigerantes e alguns sucos de frutas. 
De 4 a 8 anos de idade, quando a família passava o dia fora de casa, os pais ofereciam salgadinhos industrializados e biscoitos, pois a criança não consumia alimentos desconhecidos. Nesse período recebeu tratamento psicológico específico a SA, no entanto não houve resposta, porque VNS se apresentava indiferente ao problema.

Na consulta nutricional inicial, aos 14 anos e 8 meses de idade, observou-se nas curvas de crescimento (Figura 1 e 2) que ocorreram algumas variações na evolução da estatura e peso, porém em nenhum momento afastou-se demasiadamente da média esperada (P50) para o IMC e na estatura para idade. Encontrava-se no estágio 3 de Tanner ${ }^{4}$, ou seja, em fase anterior à velocidade máxima de crescimento.

Não foi encontrada nenhuma alteração significativa nos exames bioquímicos que justificasse a SA. Apesar disso, o paciente fazia uso de suplemento multivitamínico e mineral há três anos, prescrito pelo pediatra por causa da limitada variedade na alimentação.

\section{DISCUSSÃO}

A literatura é escassa em estudos de casos de crianças com seletividade alimentar, porém Timimi et al. ${ }^{5}$ acompanharam durante quatro anos 33 crianças seletivas, com idades entre 4 e 14 anos. Dois terços da amostra eram meninos e a minoria apresentava déficit de crescimento e ganho ponderal, assim como o paciente deste caso. As crianças eram ansiosas e apresentavam sintomas obsessivo-compulsivos com a comida e outros comportamentos não relacionadas com ela. As refeições representavam momentos de conflito, especialmente em crianças menores, e quando já não haviam muitas tentativas para mudanças de hábitos alimentares, situações observadas neste caso.

De acordo com a literatura, a amamentação poderia facilitar a aceitação de novos alimentos, visto que as características sensoriais do leite materno são influenciadas pela dieta da mãe e permite o primeiro contato da criança com sabores e odores variados ${ }^{6-8}$. Esse contato possibilitaria a melhora da aceitação de novos alimentos quando oferecidos inicialmente. $O$ curto período de aleitamento materno neste caso impossibilitou essa experiência, e até os 5 meses de idade ele só conhecia o sabor do leite artificial.

Crianças que apresentam atraso na introdução de alimentos sólidos durante o primeiro ano de vida estão mais propensas a desenvolver comportamentos seletivos ao longo da infância, sendo estes mais claramente identificados ao redor dos 7 anos de idade ${ }^{9,10}$. Neste caso houve boa aceitação na introdução das frutas, porém houve recusa de alimentos salgados sólidos até 1 ano de idade. Tal comportamento poderia predispor à SA, conforme indicam os estudos mencionados.
Maier et al. ${ }^{6}$ ressaltam que o número de exposições ao novo alimento seria mais determinante para a aceitação do que a variedade, sendo importante alternar as formas de apresentação, preparação e textura ${ }^{6-8}$. A eficácia dessa conduta pôde ser observada em crianças de cidades europeias ${ }^{7}$. Neste caso foram oferecidos vários alimentos em diversas formas de apresentação ou preparação, mas com difícil aceitação. A maneira de oferecer alimentos também foi variada, como alternar entre dar comida na boca ou deixar comer sozinho, assim como dispor os alimentos em pratos em diferentes locais da casa.

Segundo Bryant-Waugh et al. ${ }^{11}$, o quadro clínico da SA está normalmente associado ao consumo de alimentos de uma tonalidade (como branco) e de sabor muito suave (como leite, pão, macarrão etc.) ou determinada textura (pastoso ou crocante), com recusa de preparações com alimentos de texturas variadas. Em certos casos é possível observar preferência por determinadas marcas de alimentos ou mesmo a temperatura em que são servidos (frios ou quentes). Além disso, algumas crianças não toleram o odor de alimentos que não fazem parte de sua restrita lista de preferências, prejudicando seu convívio com familiares e amigos no momento das refeições. Neste caso, o paciente apresentava preferência por alimentos de cor clara, batata, biscoitos, pães e frutas, e alimentos com textura como purês que ainda não são aceitos.

De acordo com a literatura, algumas crianças mostram-se ansiosas diante de alimentos com molho ou que são fáceis para se sujar, comportamento que pode estar associado a mães obsessivas por limpeza ou que não permitiram ao filho usar as mãos para alimentar-se ${ }^{5}$. Alimentos com molho nunca foram aceitos pelo paciente, mas neste caso não se deve às atitudes inadequadas da mãe.

A família e os cuidadores são fundamentais para a formação e a estruturação dos hábitos e condutas alimentares das crianças. Também são responsáveis por promover o ambiente das primeiras experiências alimentares que podem ser decisivas para a aceitação de novos alimentos ${ }^{3,12}$. Se um ambiente é agradável e novos alimentos são oferecidos sem coação, a criança fica muito mais suscetível a desenvolver preferência por eles.

Neste caso, sempre coube à mãe a responsabilidade pela alimentação da família, e ela procurou variar a alimentação do filho, enquanto o pai pouco se envolvia na questão alimentar e, quando o fazia, era sempre impaciente e rude com o filho, tinha atitudes inadequadas, como deixar o filho sem comer quando ele se recusava a comer o que era oferecido. O paciente relata que não tinha afinidade com o pai e havia pouco diálogo entre eles.

Sabe-se que a desestruturação familiar pode influenciar a SA em algumas crianças ${ }^{13,14}$, sendo importante a conscientização do problema vivenciado, de forma a impor limites sem, contudo, perder o controle. Não podemos afir- 
mar que a família era desestruturada, contudo levava VNS ao descontrole emocional, situação que não colaborava para a melhora do quadro. É importante ressaltar a recomendação de que os pais devem ponderar suas atitudes e ambos devem participar ativamente no processo de educação alimentar.

Em alguns casos, quando a criança não supre as expectativas dos pais com relação ao consumo alimentar, o descontrole emocional que se instala coloca a criança no controle da situação, permitindo que ela mesma decida o que comer, e não seus responsáveis ${ }^{13,14}$. É importante ressaltar que crianças seletivas normalmente apresentam comportamentos fóbicos diante de alimentos que não são de sua preferência, gerando confrontos e atitudes agressivas para evitar comer o que não querem ${ }^{5}$.

A ansiedade dos familiares para ver a criança comer levaria à substituição de alimentos saudáveis por aqueles de baixo valor nutritivo, os quais normalmente fazem parte das preferências dos seletivos. Dados demonstram que 45\% dos pais gostariam de mudar os hábitos das crianças; para isso, $51 \%$ oferecem recompensas e $69 \%$ persuadem seus fiIhos $^{14,15}$. Com relação a esse tema, a mãe não permitia substituir as refeições principais por guloseimas, com exceção dos dias em que a família passava o dia fora de casa.

O prejuízo social parece ser uma característica comum no cotidiano dos indivíduos identificados com seletividade alimentar, especialmente quando a lista de alimentos aceitáveis é muito restrita. A criança pode sentir-se intimidada pelos demais e, à medida que vai crescendo, seu constrangimento sobre sua forma de alimentar-se a torna muito reservada ${ }^{11}$. Esse foi um dos motivos que levaram o paciente a procurar tratamento.

\section{Evolução do tratamento nutricional}

Na primeira consulta nutricional, foi discutido com o paciente e sua mãe o conceito de neofobia alimentar, para que ele não tenha expectativa de ter sucesso na primeira tentativa de experimentar um novo alimento.

Nas consultas que se seguiram, o paciente sempre vinha acompanhado da mãe, e esta e o paciente eram orientados quanto à introdução de um novo alimento, variando a textura e o preparo. O paciente era orientado a fazer o diário alimentar, para que se pudesse discutir em cada consulta os alimentos que experimentou, apresentando os que aprovou e os que não aprovou.

O paciente passou por 10 consultas nutricionais durante 10 meses. A introdução de novos alimentos foi estimulada a cada consulta, e a receptividade do paciente foi surpreendente, com aceitação de vários alimentos na primeira tentativa, que ocorreu depois da segunda consulta. Vale salientar que essa resposta positiva precoce se deu devido à aceitação por parte do paciente do diagnóstico de SA e à conscientização das consequências desse transtorno em suas relações sociais.
A evolução positiva quanto à qualidade nutricional e à variedade pode ser observada na tabela 1.

Conforme avaliação inicial (Tabela 1), identificou-se um déficit no valor calórico total da dieta consumida em relação à sua necessidade energética diária. Em relação aos macronutrientes (proteínas, gorduras e carboidratos), observa-se que estes estavam adequados, o que não foi observado entre os micronutrientes ( $\mathrm{Ca}$, Fe e vitamina $\mathrm{A}$ ), que se encontravam abaixo do ideal para a faixa etária. Ao longo do tratamento, verificou-se que o paciente conseguiu aumentar seu consumo energético, chegando ao que é recomendado para sua idade. Os macronutrientes não sofreram modificações relevantes, por outro lado houve aumento evidente no consumo de todos micronutrientes, apesar de somente o cálcio não ter alcançado o valor de referência. Estudos nacionais demonstram que o consumo inadequado de cálcio é muito frequente em adolescentes saudáveis ${ }^{16,17}$. Os resultados, portanto, indicam que o paciente atualmente apresenta um perfil nutricional semelhante ao de jovens de sua idade e que as deficiências nutricionais apresentadas inicialmente na dieta foram corrigidas.

Suas primeiras experiências com novos alimentos foram positivas. Pôde-se observar na tabela 2 que VNS foi exposto a 71 tipos de alimentos e/ou preparações no percurso do tratamento e, desse total, cerca de 65\% $(n=46)$ foram aceitos e introduzidos na sua rotina alimentar. Diante disso, pode-se afirmar que o paciente vem apresentando boa resposta à terapia nutricional, com receptividade para incluir novos alimentos.

Quanto ao seu estado nutricional, pôde-se observar (Figura 1) que a seletividade não afetou o crescimento, mantendo a estatura dentro da normalidade, dos 9 aos 14 anos. No entanto, verificou-se uma variação de peso ao longo dos anos, que refletiu em períodos de aparente sobrepeso dos 9 aos 13 anos, mas que se normalizou após essa idade, provavelmente por causa da fase do estirão em que o paciente se encontra (Figura 2). Esses dados confirmam o que a literatura aponta sobre o crescimento das crianças identificadas com SA, o qual não necessariamente fica comprometido ${ }^{18}$. A restrição na variedade de alimentos consumidos na infância não necessariamente tem impacto no estado nutricional, uma vez que muitas vezes a criança consome uma quantidade maior desses alimentos específicos, sendo suficiente para suprir suas necessidades energéticas.

Nas figuras 1 e 2, pode-se observar que houve estabilização em relação ao crescimento, pois o paciente se manteve no P50. Passados 10 meses de tratamento, observa-se que o paciente realmente incluiu os alimentos de que gostou, incorporando-os na sua rotina alimentar. Persiste ainda a resistência em aceitar preparações com consistência pastosa (purê de batatas) e com molho (feijão, estrogonofe). O aumento da variedade de alimentos permitiu melhorar suas relações sociais e se envolver em atividades como almoçar com os amigos da escola. 
Tabela 1. Evolução do padrão nutricional nas consultas

\begin{tabular}{|c|c|c|c|c|c|c|c|c|c|}
\hline Padrão nutricional* & $31 / 05$ & $14 / 06$ & $28 / 06$ & 09/08 & $30 / 08$ & $27 / 09$ & $18 / 10$ & 08/11 & $\begin{array}{l}\text { Valores de } \\
\text { referência** }\end{array}$ \\
\hline Valor calórico (kcal) & 2.200 & 2.605 & Não fez diário & 2.721 & 2.750 & 2.972 & 3.006 & 3.237 & 2.932 \\
\hline \% proteína & 13 & 13,9 & & 12,4 & 14,3 & 14,1 & 13,8 & 14,31 & 10 a 30 \\
\hline$\%$ carboidrato & 61 & 58,9 & & 64,2 & 56,9 & 54,0 & 57,5 & 63,38 & 45 a 65 \\
\hline$\%$ gordura & 26 & 28 & & 23,4 & 28,7 & 31,8 & 28,7 & 22,31 & 25 a 35 \\
\hline Cálcio (mg) & 155,8 & 159,2 & & 296,0 & 189,9 & 247,1 & 603,1 & 577,4 & 1.300 \\
\hline Ferro (mg) & 4,6 & 6,4 & & 8,1 & 12,4 & 13,5 & 13,4 & 18,8 & 11 \\
\hline Vitamina A $(\mu \mathrm{g})$ & 114 & 488,8 & & 437,6 & 480,4 & 536,7 & 974,6 & 778,0 & 900 \\
\hline
\end{tabular}

* Os valores nutricionais foram calculados pelo programa de análise nutricional (AVANUTRI).

** Valores recomendados pelo National Research Council (NRC) for energy e Standing Committee on the Scientific Evaluation of Dietary Reference Intakes (DRI Committee) para valor calórico, porcentagem de proteínas, carboidratos, gordura e consumo de cálcio, ferro e vitamina A.

Tabela 2. Evolução da introdução dos alimentos nas consultas

\begin{tabular}{|c|c|c|c|c|c|c|c|}
\hline & $14 / 06$ & $28 / 06$ & 09/08 & $30 / 08$ & $27 / 09$ & $18 / 10$ & 08/11 \\
\hline $\begin{array}{l}\text { Alimentos } \\
\text { introduzidos e } \\
\text { aceitos }\end{array}$ & $\begin{array}{c}\text { Cenoura ralada } \\
\text { Rúcula } \\
\text { Camarão empanado } \\
\text { Salsicha } \\
\text { Filé de frango } \\
\text { Ovo frito } \\
\text { Pizza de calabresa }\end{array}$ & $\begin{array}{l}\text { Beterraba } \\
\text { Agrião } \\
\text { Medalhão } \\
\text { bovino } \\
\text { Batata } \\
\text { assada } \\
\text { Polenta }\end{array}$ & $\begin{array}{l}\text { Salada de feijão } \\
\text { branco } \\
\text { Salada de espinafre } \\
\text { Peixe empanado } \\
\text { Macarrão }\end{array}$ & $\begin{array}{c}\text { Arroz de forno com frango } \\
\text { e milho } \\
\text { Couve-flor empanada frita } \\
\text { Macarrão ao pesto } \\
\text { Lentilha sem caldo } \\
\text { Almôndega bovina } \\
\text { Salmão cru e grelhado } \\
\text { Rolinho primavera } \\
\text { Uva } \\
\text { Pera } \\
\text { Abacaxi }\end{array}$ & $\begin{array}{l}\text { Risoto de camarão } \\
\text { Macarrão alho e óleo } \\
\text { Salada de lentilha e } \\
\text { cenoura ralada } \\
\text { Bolinho de espinafre } \\
\text { Bolinho de arroz } \\
\text { Pão de queijo } \\
\text { Torrada } \\
\text { Manteiga } \\
\text { Requeijão } \\
\text { Esfirra de carne }\end{array}$ & $\begin{array}{c}\text { Salame } \\
\text { Peito de peru } \\
\text { Costelinha de } \\
\text { porco } \\
\text { Acelga } \\
\text { Morango }\end{array}$ & $\begin{array}{c}\text { Espaguete ao molho } \\
\text { de calabresa } \\
\text { Arroz } 7 \text { grãos } \\
\text { Couve refogada } \\
\text { Carne de churrasco } \\
\text { (fraldinha) } \\
\text { Bisteca de porco }\end{array}$ \\
\hline $\begin{array}{l}\text { Alimentos } \\
\text { introduzidos e } \\
\text { não aceitos }\end{array}$ & Bife (paillard fino) & $\begin{array}{l}\text { Brócolis } \\
\text { Pepino } \\
\text { Laranja } \\
\text { Mexerica }\end{array}$ & $\begin{array}{c}\text { Rabanete } \\
\text { Purê de batata }\end{array}$ & $\begin{array}{l}\text { Couve-flor cozida } \\
\text { Vagem } \\
\text { Mandioquinha } \\
\text { Mandioca frita } \\
\text { Arroz branco }\end{array}$ & $\begin{array}{l}\text { Patê de atum com } \\
\text { maionese } \\
\text { Geleia }\end{array}$ & $\begin{array}{l}\text { Tomate seco } \\
\text { Abobrinha } \\
\text { refogada } \\
\text { Capelleti } \\
\text { Pimentão } \\
\text { Manga } \\
\text { Amora }\end{array}$ & $\begin{array}{c}\text { Ameixa } \\
\text { Abóbora refogada } \\
\text { Espinafre refogado } \\
\text { Palmito } \\
\text { Carne seca }\end{array}$ \\
\hline
\end{tabular}

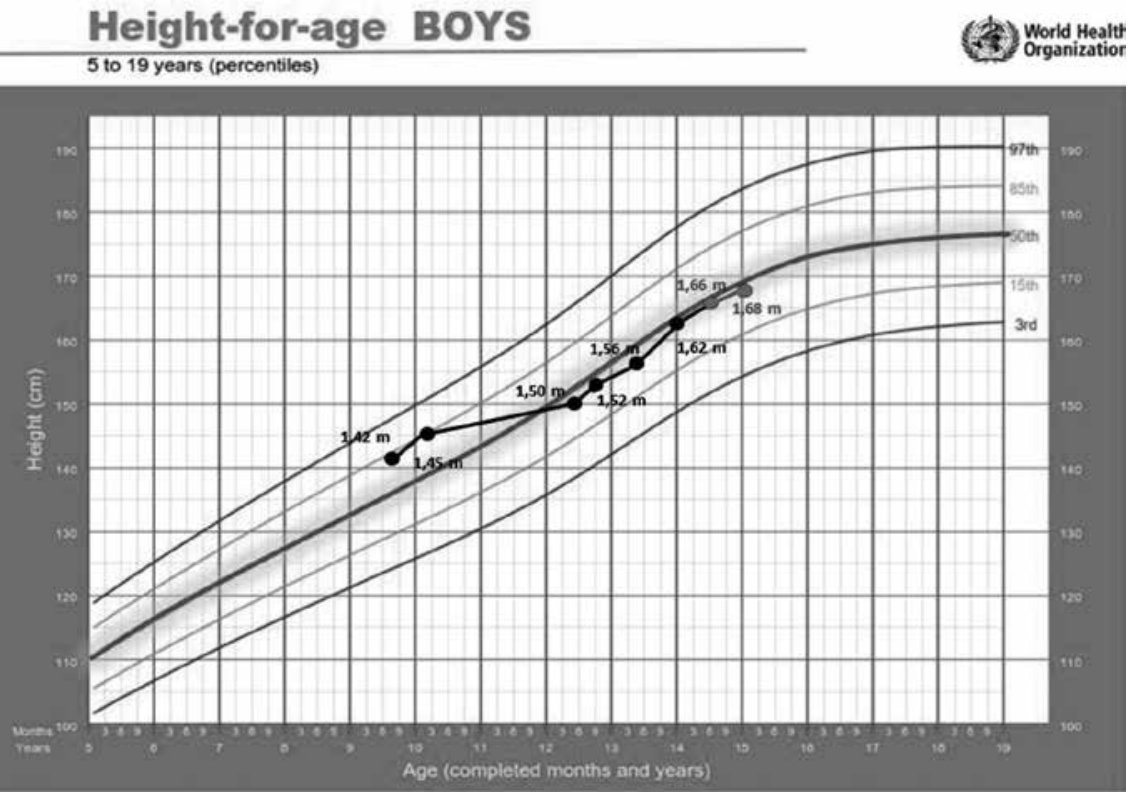

Figura 1. Curvas de referência para estatura para idade e sexo de acordo com as normas da Organização Mundial da Saúde (2006 e 2007). 


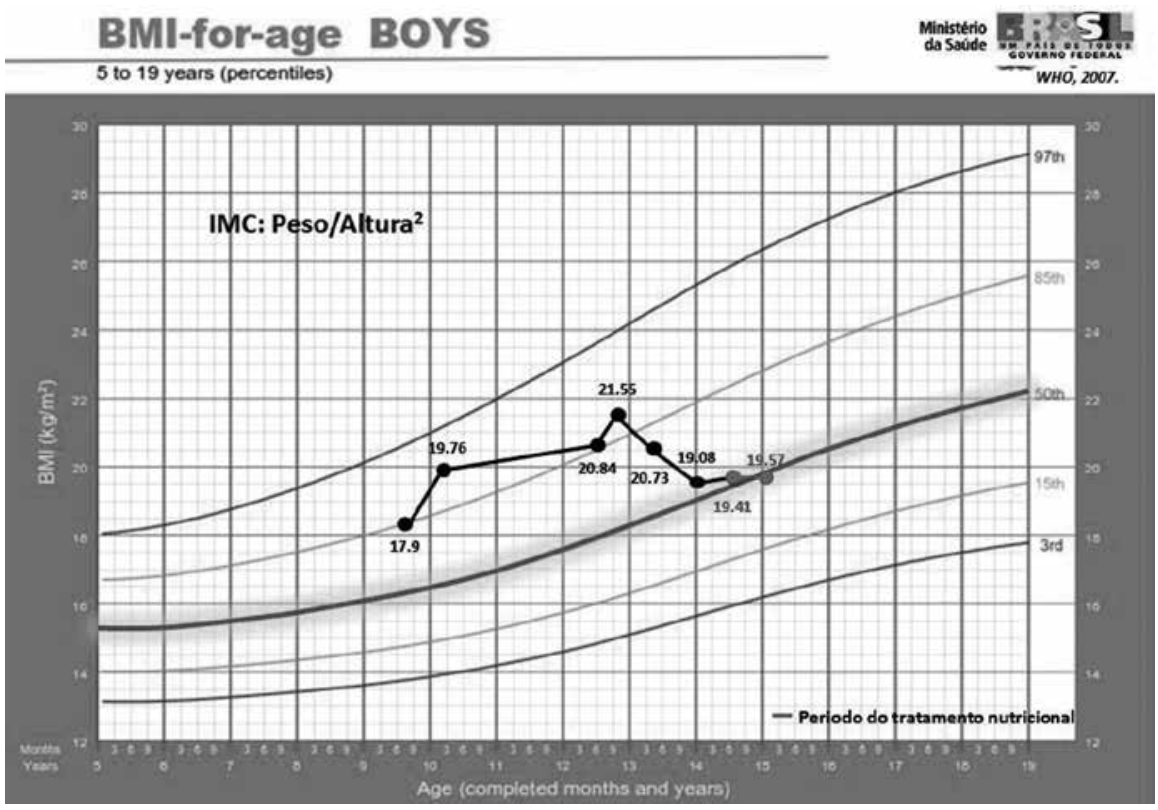

Figura 2. Curvas de referência para índice de massa corporal para idade e sexo de acordo com as normas da Organização Mundial da Saúde (2006 e 2007).

\section{CONCLUSÃO}

A fim de minimizar as consequências da má alimentação promovida pela seletividade, é importante a participação ativa dos pais e cuidadores na identificação dos sinais e sintomas característicos da SA. O tratamento precoce evita as consequências de carências nutricionais e possibilita o crescimento e o desenvolvimento adequado, garantindo, assim, melhor prognóstico.

\section{CONTRIBUIÇÕES INDIVIDUAIS}

Ana Beatriz de Mello Sampaio - Participou da coleta de dados, concepção, análise e interpretação dos dados e da elaboração do artigo.

Ruth Bartelli Grigolon, Ana Maria Roma, Leticia Enrique Pereira - Participaram do desenho do estudo, interpretação dos dados e elaboração do artigo.

Karin Louise Lenz Dunker, Thais Lourenço Nogueira Participaram da coordenação, concepção, desenho do estudo, análise e interpretação dos dados e elaboração do artigo.

\section{REFERÊNCIAS}

1. Nicholls D, Bryant-Waugh R. Eating disorders of infancy and childhood: definition, symptomatology, epidemiology, and comorbidity. Child Adolesc Psychiatric Clin N Am. 2008;18:17-30.
2. Jacobi C, Schmitz G, Agras WS. Is picky eating an eating disorder? Int J Eat Disord. 2008:41(7):626-34

3. Carruth BR, Ziegler PJ, Gordon A, Barr SI. Prevalence of picky eaters among infants and toddlers and their caregivers' decisions about offering a new food. J Am Diet Assoc. 2004;104(S1):557-64.

4. Tanner JM. Growth at adolescence. Oxford: Blackwell; 1962.

5. Timimi S, Douglas J, Tsiftsopoulou K. Selective eaters: a retrospective case note study. Child Care Health Dev. 1997;23(3):265-78.

6. Maier AS, Chabanet C, Schaal B, Leathwood PD, Issanchou SN. Breastfeeding and experience with variety early in weaning increase infants' acceptance of new foods for up to two months. Clin Nutr. 2008;27(6):849-57.

7. Maier A, Chabanet C, Schaal B, Leathwood P, Issanchou S. Food-related sensory experience from birth through weaning: contrasted patterns in two nearby European regions. Appetite. 2007:49(2):429-40.

8. Northstone $K$, Emmett $P$, Nethersole F. The effect of age of introduction to lumpy solids on foods eaten and reported feeding difficulties at 6 and 15 months. J Hum Nutr Diet. 2001;14(1):43-54.

9. Shim JE, Kim J, Mathai RA. Associations of infant feeding practices and picky eating behaviors of preschool children. J Am Diet Assoc. 2011;111(9):1363-8.

10. Pearson N, Biddle SJH, Gorely T. Family correlates of fruit and vegetable consumption in children and adolescents: a systematic review. Public Health Nutr. 2009;12(2):267-83.

11. Bryant-Waugh R, Markham L, Kreipe RE, Walsh BT. Feeding and eating disorders in childhood. Int J Eat Disord. 2010;43(2):98-111.

12. Birch $L L$, Davison KK. Family environmental factors influencing the developing behavioral controls of food intake and childhood overweight. Pediatr Clin North Am. 2001;48(4):893907.

13. Galloway AT, Lee Y, Birch LL. Predictors and consequences of food neophobia and pickiness in young girls. J Am Diet Assoc. 2003;103(6):692-8.

14. Skinner JD, Carruth BR, Wendy B, Ziegler PJ. Children's food preferences: a longitudinal analysis. J Am Diet Assoc. 2002;102(11):1638-47. 
15. Reifsnider E, Allan J, Percy M. Mothers' explanatory models of lack of child growth. Public Health Nurs. 2000;17(6):434-42.

16. Leal GVS, Philippi ST, Matsudo SMM, Toassa EC. Consumo alimentar e padrão de refeições de adolescentes, São Paulo, Brasil. Rev Bras Epidemiol. 2010;13(3):457-67.

17. Miguel VP, Resende CMM, Del Ciampo LA, Daneluzzi JC, Ricco RG, Ferraz IS, et al. Ingestão dietética, avaliação antropométrica e composição corporal de adolescentes atendidos em um Centro de Atenção Primária à Saúde em Ribeirão Preto, São Paulo, Brasil. Medicina (Ribeirão Preto). 2011;44(3):267-75.

18. Reau NR, Senturia YD, Lebailly SA, Chrisoffel KK. Infant and toddler feeding patterns and problems: normative data and a new direction. Pediatric Practice Research Group. J Dev Behav Pediatr. 1996;17(3):149-53. 